\title{
Search for Long Lived Particles that Stop in CMS
}

\section{Jim Brooke on behalf of the CMS Collaboration* \\ University of Bristol}

E-mail: jim.brooke@cern.ch

\begin{abstract}
The CMS collaboration will search for long-lived particles that have become stopped by the CMS detector. The decay of such particles during time intervals where there are no $p p$ collisions would be a signal with low and easily measured backgrounds, coming from instrumental effects and cosmic rays. The search includes periods between gaps in the LHC bunch structure, and periods between dumping the beam and re-injection. Stopped particle decays will be collected using dedicated calorimeter triggers. For models such as split-supersymmetry gluinos, the relatively large cross-section combined with the relatively good stopping power of CMS, yields a significant number of triggerable decays. As the LHC instantaneous luminosity approaches $10^{32} \mathrm{~cm}^{-2} \mathrm{~s}^{-1}$, at $\sqrt{s}=10 \mathrm{TeV}$, a 5 sigma significance can be established in a matter of days.
\end{abstract}

XXth Hadron Collider Physics Symposium

November 16 - 20, 2009

Evian, France

* Speaker. 


\section{Introduction}

A number of new physics scenarios beyond the Standard Model predict the existence of new heavy quasi-stable charged particles [1]. The existence of such particles may also explain the discrepancy in ${ }^{7} \mathrm{Li}$ abundance between big-bang nucleosynthesis predictions and observation [2]. If heavy stable charged particles are produced at the LHC, they may lose energy traversing the detector and come to rest. The subsequent decay of these particles may occur at any time, and can be observed during periods when no $p p$ collisions are occurring. The only backgrounds during these periods are due to cosmic rays and instrumental effects. Such signals will be searched for using the CMS detector [3], with the methodology presented here.

\section{Signal Simulation}

In order to study stopped particles, a custom simulation treatment was developed for particles with long lifetimes. The simulation comprises three stages. In Stage 1, the passage of stable particles through the CMS detector is simulated, noting if and where the particle has come to rest. This process is repeated many times to obtain a 3D probability density function for the stopping of such a particle at an arbitrary location within the CMS detector 1 . The highest density of stopped particles is found in the hadronic calorimeter (HCAL). In Stage 2, this "stopping map" is sampled to obtain a probable stopping location. A particle is generated at rest at this position, and then decayed instantaneously, allowing the detector response and reconstruction efficiency to be simulated. Stage 3 simulates the time of this decay, relative to the LHC beam clock, for a given particle lifetime.
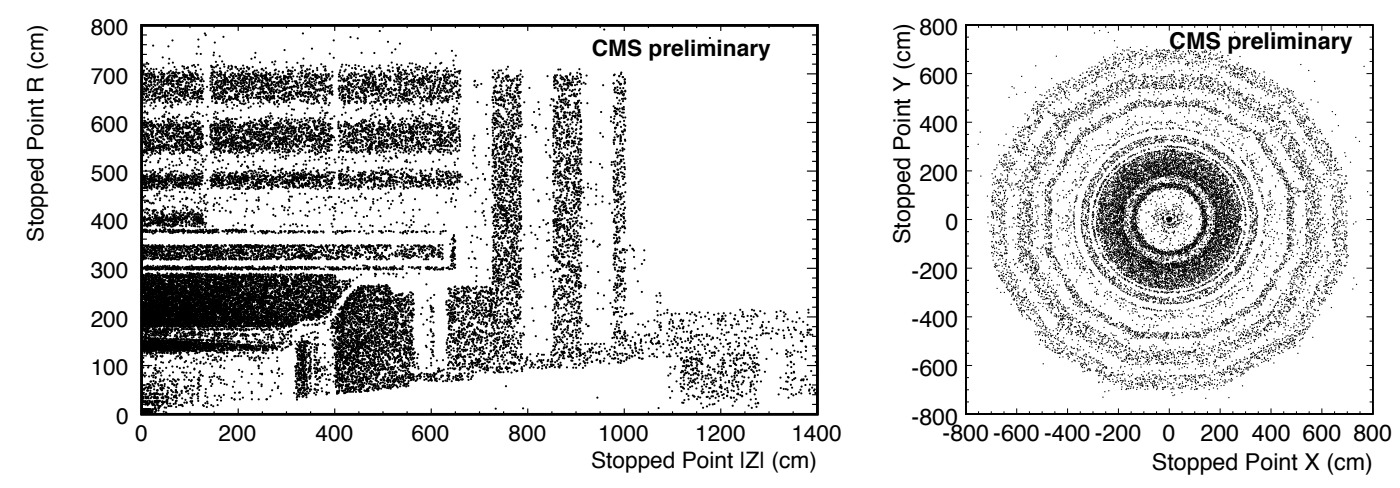

Figure 1: R-hadron stopping points for $m_{\tilde{g}}=300 \mathrm{GeV}$, and $\sqrt{s}=10 \mathrm{TeV}$. In the $R-|z|$ plane (Left) and for the barrel only in $x-y$ plane (Right).

\section{Trigger Selection and Offline Analysis}

To trigger on decays of stopped particles, a standard Level-1 calorimeter jet candidate is required, above an $E_{T}$ threshold of $10 \mathrm{GeV}$. Beam position and timing monitors are used to select events occurring during periods of no collisions. These devices are located up and downstream of CMS, and produce signals in time with passing bunches. A coincidence of signals from both 
monitors is used as a veto at Level-1, to prevent triggering on collision products. In the higher-level software trigger, a standard iterative cone jet algorithm is used to reconstruct jets in HCAL; a single jet is required, above an energy threshold of $20 \mathrm{GeV}$. The trigger will be run during periods when beam is circulating, to trigger on gaps between bunches and between dumping and re-injection of the beam, to maximise the range of lifetimes that can be detected.

The main backgrounds to the signal are instrumental noise and cosmic rays. Both have been studied in CMS data taken before LHC startup. Instrumental noise is rejected using cuts based on jet topology and the calorimeter signal time-profile. The cosmic ray background is rejected by vetoing on reconstructed muons. These selections were tuned on the pre-LHC background data and signal Monte-Carlo samples. The background rate, measured with the pre-LHC data, is $3.9 \pm 0.9 \times 10^{-4} \mathrm{~Hz}$, and the overall efficiency for a split-SUSY signal $\left(m_{\tilde{g}}=300 \mathrm{GeV}, m_{\tilde{\chi}^{0}}=100\right.$ $\mathrm{GeV}$ ) is $16.4 \%$ of all R-hadrons that stop in the CMS detector.

\section{Split-Supersymmetry Discovery Potential}

Given the background rate measured with pre-LHC data, the sensitivity to a signal can be estimated. Figure 2 shows the sensitivity to a split-SUSY gluino signal (with a cross-section of $\sigma=0.5 \mathrm{nb}$ ) for a simple counting experiment, combining beam-gap and inter-fill periods. LHC fills are assumed to last 12 hours, with a 12 hour period between fills. Several counting experiments are performed, for a range of sampling times, to ensure sensitivity to a range of lifetimes without integrating excessive background. At an instantaneous luminosity of $10^{32} \mathrm{~cm}^{-2} \mathrm{~s}^{-1}$, a discovery can be achieved with data from just a few days running. The search is less sensitive at lower instantaneous luminosities, but a 95\% CL exclusion limit can be set with a few days running at $L=10^{31}$ $\mathrm{cm}^{-2} \mathrm{~s}^{-1}$. The sensitivity as a function of gluino mass is shown in Figure 2 (Left), the dominant effect on the sensitivity being the gluino production cross-section, which falls steeply. The generic search for stopped particles is minimally exposed to substantial systematic uncertainties. However, the interpretation in terms of a given model, such as split-SUSY presented here, incorporates systematic uncertainties with the effect on sensitivity show in Figure 3 (Right). The dominant uncertainty is that associated with the R-hadron "cloud model" of nuclear interactions [4], and hence the probability of a produced gluino to stop in the detector.

\section{Summary}

Long lived particles that stop in the CMS detector have been simulated, allowing a search strategy to be developed. A novel calorimeter trigger allows recording of stopped particle decays that occur asynchronously with $p p$ collisions. For models with relatively large cross-sections $(\sim 1 \mathrm{nb})$, a 5 sigma discover can be made in a matter of days with an instantaneous luminosity of $10^{32} \mathrm{~cm}^{-2}$ at $\sqrt{s}=10 \mathrm{TeV}$. This sensitivity improves greatly on that achieved in previous experiments [5], in part because of the novel triggering strategy that has been implemented.

\section{References}

[1] M. Fairbairn, A. C. Kraan, D. A. Milstead, T. Sjostrand, P. Z. Skands and T. Sloan, Phys. Rept. 438 (2007) 1 

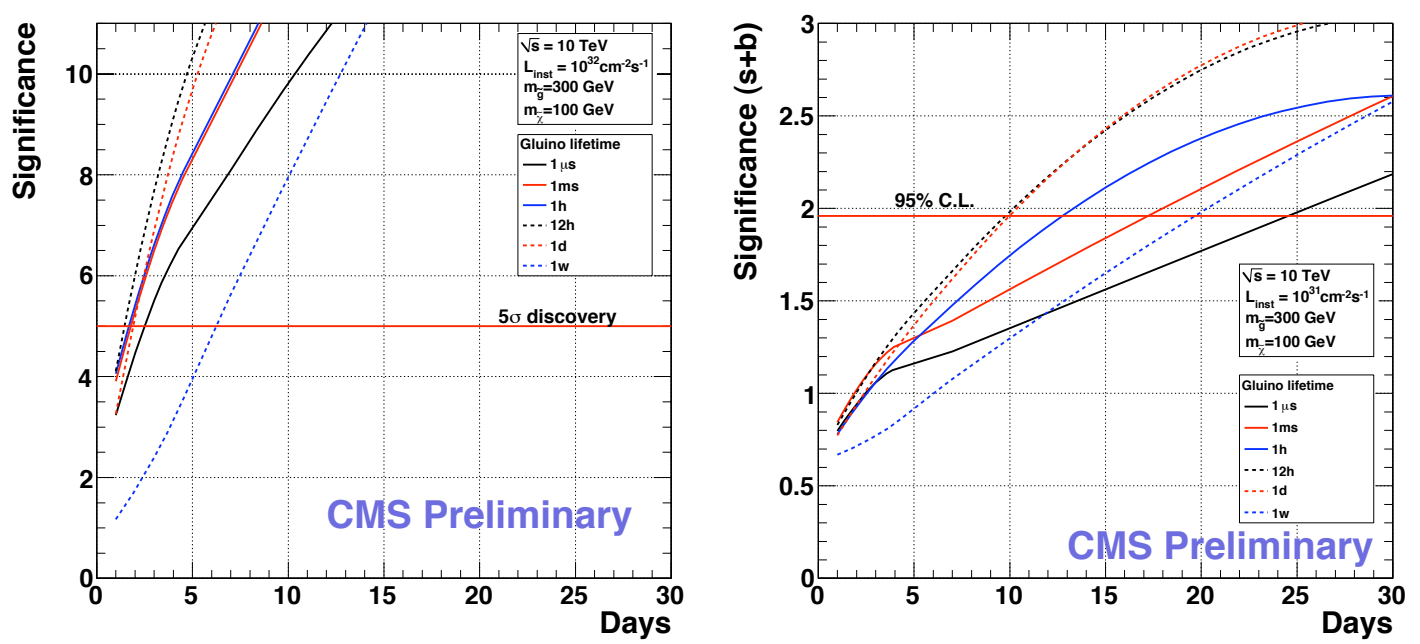

Figure 2: Signal significance as a function of data-taking time, for a counting experiment combining both beam gaps and interfill periods for a range of gluino lifetimes, at instantaneous luminosities of $10^{32} \mathrm{~cm}^{-2}$ $\mathrm{s}^{-1}$ (Left) and $10^{31} \mathrm{~cm}^{-2} \mathrm{~s}^{-1}$ (Right).
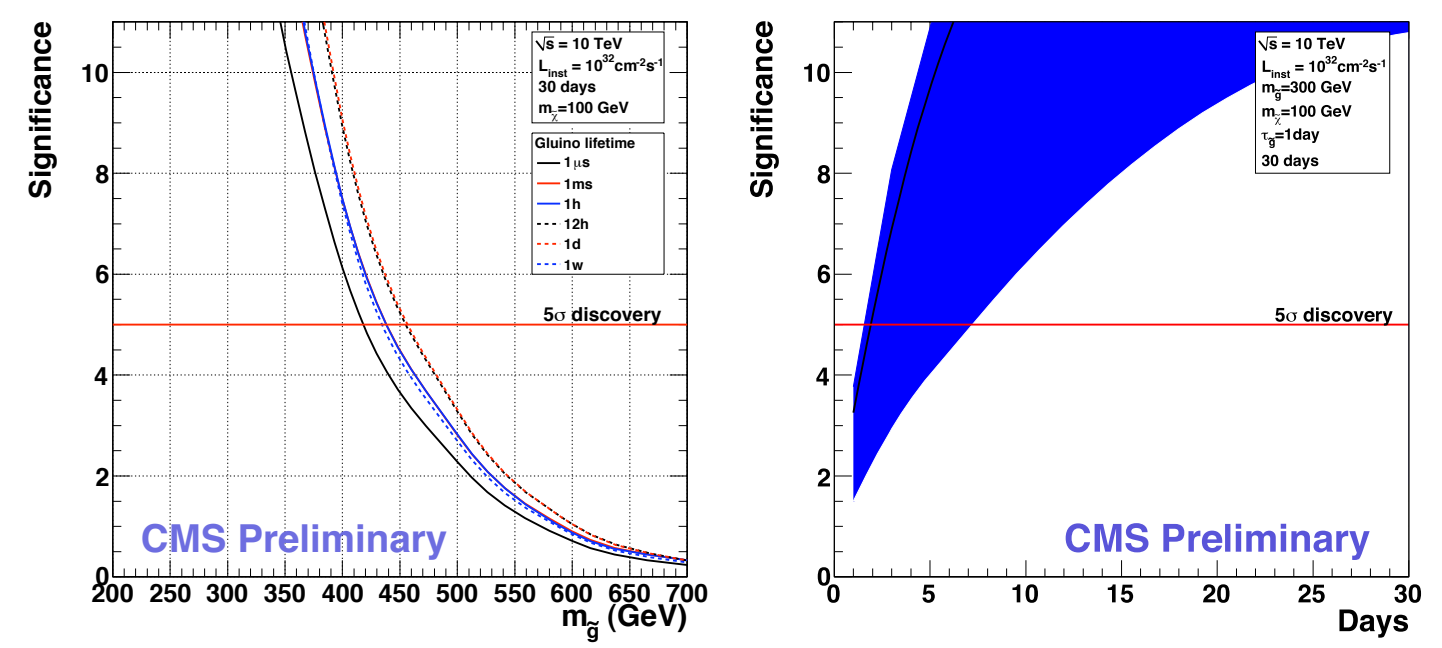

Figure 3: Signal significance achievable after 30 days running, as a function of $m_{\tilde{g}}$ from a counting experiment using both beam-gap and inter-fill periods (Left). Effect of systematic uncertainty on the discovery potential for a gluino with $m_{\tilde{g}}=300 \mathrm{GeV}$, indicated by the blue band (Right).

[2] T. Jittoh, K. Kohri, M. Koike, J. Sato, T. Shimomura and M. Yamanaka, Phys. Rev. D 78 (2008) 055007

[3] R. Adolphi et al. [CMS Collaboration], JINST 0803 (2008) S08004 [JINST 3 (2008) S08004].

[4] R. Mackeprang and A. Rizzi, Eur. Phys. J. C 50 (2007) 353

[5] V. M. Abazov et al. [D0 Collaboration], Phys. Rev. Lett. 102 (2009) 161802 